\title{
Exploiting Fitness Apps for Sustainable Mobility - Challenges Deploying the GoEco! App
}

\author{
Dominik Bucher \\ Institute of Cartography \\ and Geoinformation \\ ETH Zurich \\ Stefano-Franscini-Platz 5 \\ 8093 Zurich, Switzerland \\ dobucher@ethz.ch
}

\author{
Francesca Cellina \\ Insitute for Applied Sustainability \\ to the Built Environment \\ SUPSI \\ via Trevano \\ 6952 Canobbio, Switzerland \\ francesca.cellina@supsi.ch
}

\author{
Francesca Mangili \\ Dalle Molle Institute for \\ Artificial Intelligence \\ USI - SUPSI \\ Galleria 2 \\ 6928 Manno, Switzerland \\ francesca@idsia.ch
}

\author{
Martin Raubal \\ Institute of Cartography \\ and Geoinformation \\ ETH Zurich \\ Stefano-Franscini-Platz 5 \\ 8093 Zurich, Switzerland \\ mraubal@ethz.ch
}

\author{
Roman Rudel \\ Insitute for Applied Sustainability \\ to the Built Environment \\ SUPSI \\ via Trevano \\ 6952 Canobbio, Switzerland \\ roman.rudel@supsi.ch
}

\author{
Andrea Emilio Rizzoli \\ Dalle Molle Institute for \\ Artificial Intelligence \\ USI - SUPSI \\ Galleria 2 \\ 6928 Manno, Switzerland \\ andrea@idsia.ch
}

\author{
Omar Elabed \\ Laboratory of \\ Visual Culture \\ SUPSI \\ via Trevano \\ 6952 Canobbio, Switzerland \\ omar.elabed@supsi.ch
}

\begin{abstract}
The large interest in analyzing one's own fitness led to the development of more and more powerful smartphone applications. Most are capable of tracking a user's position and mode of locomotion, data that do not only reflect personal health, but also mobility choices. A large field of research is concerned with mobility analysis and planning for a variety of reasons, including sustainable transport. Collecting data on mobility behavior using fitness tracker apps is a tempting choice, because they include many of the desired functions, most people own a smartphone and installing a fitness tracker is quick and convenient. However, as their original focus is on measuring fitness behavior, there are a number of difficulties in their usage for mobility tracking. In this paper we denote the various challenges we faced when deploying GoEco! Tracker (an app using the Moves ${ }^{\circledR}$ fitness tracker to collect mobility measurements), and provide an analysis on how to best overcome them. Finally, we summarize findings after one month of large scale testing with a few hundred users within the GoEco! living lab performed in Switzerland.
\end{abstract}

Index Terms-Mobility tracking; Fitness App; Transport mode

\section{INTRODUCTION}

Mobility data are used in several fields of research, most notably transport analysis and planning, e.g., to plan transport infrastructures of cities. They are also required to analyze energy consumption caused by mobility needs and to find ways to foster more sustainable mobility behavior. To collect the required data, a variety of options are available.

On a large scale, mobility data are usually recorded on a rather inaccurate level using very time consuming methods. A prominent example is micro census collection, where home and work addresses of users and their most frequently taken routes are collected by means of in-person or phone interviews (e.g., the Swiss micro census on mobility and transport [1]).
On a smaller scale, a widely chosen approach is to ask users to write travel diaries, i.e., logs containing the routes travelled and transport modes chosen. These daily updated logs are very detailed, but the process places large burdens on the participants and makes it prone to errors [2]: people skip filling in the diary, forget about their routes, the exact paths taken, or the modes of transport used. With the increased availability of smartphones (e.g., in Switzerland, in 2014, there were approx. 140 mobile telephone subscriptions per 100 citizens [3]), a new, unobtrusive way of collecting mobility data has emerged: as mobile phones feature more and more powerful hardware and a broad range of sensors, they have become an easy means for mobility data collection.

Availability of new possibilities for automatic mobility tracking inspired our GoEco! project 1 , which aims at investigating if eco-feedback and social interactions (social comparison and peer pressure), automatically provided via information and communication technologies (ICT), can be effective in fostering long term changes in personal mobility behavior and reducing car use.

In this paper we present the approach for mobility data tracking we adopted within the GoEco! project, when developing the GoECo! Tracker app. Even though a large body of literature concerns systems and algorithms to accurately identify mobility patterns using smartphone sensors, only a few prototypical apps for automatic mobility tracking are available (e.g., PEACOX [4], Mobidot [5], Studio mobilità [6], or Modalyzer ${ }^{2}$ and it remains challenging for a small team of

\footnotetext{
${ }^{1}$ http://www.goeco-project.ch
}

2 https://www.modalyzer.com 
researchers to develop a tracking app for a large-scale study. The work required to support the variety of devices, the finetuning to keep energy consumption within acceptable ranges and the selection of appropriate activity and route recognition algorithms make it difficult to build such an app. A viable alternative is the use of already existing applications, such as location-enabled fitness trackers. The benefit is clear: there is no need to spend resources on the development of the tracking part, but instead researchers can focus on the analysis of data or on other aspects, such as using information and communication technology to support behavior change [7].

Following these considerations, in order to have a base for setting up the automatic mobility tracking, in GoEco! we opted for exploiting the fitness tracker app Moves ${ }^{\circledR}$. Here we discuss challenges we faced when setting up the GoEco! Tracker application, strategies on how to refine collected data, what accuracy can be expected and what kind of analyses can be performed with it.

\section{Mobility Monitoring For SUStainability}

Recently, a few research projects explored possibilities to exploit eco-feedback information to foster sustainable behaviour. Studies from other domains (such as household electricity consumption, cf. [8]) reported energy saving effects in the range of $5-12 \%$, by using ICT to collect data and provide feedback [9]. A few smartphone-based mobility tracking experiences aimed at promoting sustainable mobility choices were already performed in the last years (e.g., [10],[11],[12], [13]). Many papers however only report qualitative findings and lack long study periods and a sufficiently large sample of participants [14]. UbiGreen, for example, looked at a sample of 14 participants over the course of a month, partially caused by the fact, that participants had to be given specialized hardware for the experiment [10]. With GoEco!, we look at a large sample of a few hundred participants over the course of approximately a year. Participants are recruited on a voluntary basis and live in the Swiss cantons of Zurich and Ticino. We involve them in three mobility tracking periods: in a first one-month monitoring period (Spring 2016) we track their movements to identify baseline mobility patterns; in a second three-months period (Autumn 2016) we continue tracking movements and also use "gamification" [15] elements to challenge them to modify their mobility patterns; the final period (Spring 2017) is used to study long-term effects. Comparing the individual mobility patterns of the three periods allows us to assess the overall effectiveness of the GoEco! approach. The first challenge we face in GoEco!, therefore, is to find a way to automatically and efficiently identify the baseline mobility patterns of a large scale of users. It needs to be noted, that surveillance and behavior change are heavily debated topics (cf. [16], [17]), which we will only touch upon in the context of this paper.

\section{Large Scale Automatic Mobility Monitoring}

During our assessment of viable tracking options for GoEco!, we performed an overview of the most effective approaches for large scale and automatic mobility monitoring.

A large number of studies has focused on the recording and analysis of mobility and activity data using smartphones, usually by utilizing location (GPS) and accelerometer data ([18], [19], [20], [21], [22], [23], [24], [25], [26]). Issues such as suitability of sensors for activity recognition [27], accuracy of transport mode classification [28], [29] and energy consumption of the app [30] are well researched areas. Many of these implementations promise high recognition accuracy, up to approximately 95\% (cf. [21], [31], [27]). In modern smartphones, various algorithms have already been directly implemented in hardware and run on dedicated processors in order to save energy (cf. the M7/8/9 coprocessors in Apple devices). Nonetheless, the implementation of such algorithms across a wide range of devices remains challenging. While many studies collect data using diaries and surveys [32], there are a few which build their own mobility trackers [12], or do a mixture of both, where users can refine certain activities [10]. Another commonly found approach is to use built-in sensors of modern cars, or deploying sensors to taxi and other commercial fleets (e.g., [33]). However, there is always a large effort in developing, testing and supporting applications, which often leads to small sample sizes. In the case of GoEco!, we try to capture a large and diverse sample of the population, testing our hypotheses in a so-called "living lab" [34], a data collection method related to citizen science [35]. A reason for using a living lab is the diversity of people, which facilitates generalization [36]. Overall, in fact, approximately 400 people are actively participating in the living lab. However, high diversity of users also means a large diversity of smartphones and operating systems, which further challenges us.

To face the complexity of the tracking problem, we opted for the following approach: using an existing fitness tracker app, we build an addition on top, which refines data and allows users to interact with them. A few apps are suited for such an approach (for instance, the ultimately used tracker app Moves lists around one hundred officially approved applications that build upon its tracking system ${ }^{3}$, however they are not aimed at tracking mobility patterns but are instead focusing on the fitness performances of the users.

\section{ChOICE OF The TRACKER APP}

Since there is a broad range of trackers available (mostly from the fitness domain), a careful evaluation with respect to mobility tracking is necessary. Bauer [37] notes aspects that have to be considered when using a fitness app for route tracking: accuracy of positioning, rate of GPS points recording, inclusion of map matching algorithms, user interface and experience and data export facilities. Other considerable aspects include user ratings, reliability, quality, scope of information, aesthetics, recommendations and professional expertise [38]. Millington [39] performs an in-depth study of health and fitness apps, considering not only tracking, but

\footnotetext{
$\sqrt[3]{\text { https://apps.moves-app.com }}$
} 
overall interaction with the application and possibilities to share and link performance to "like-minded" consumers.

In our case, the choice between available tracking apps is not influenced by app usability from the user perspective: users would in fact only be requested to interact with our additional app, provided that the fitness tracker app is running in the background and it is recording the routes traveled. According to our previous experience in building mobility tracking apps [40], essential requirements for mobility tracking are:

1) reasonable accuracy of recorded GPS points (it should be possible to reconstruct a close approximation to the actual route taken by applying map matching algorithms);

2) capability to automatically identify that transport activities are taking place, without any indication by the user (no need to activate and deactivate the GPS tracking functionality);

3) availability of pre-processing algorithms able to organize GPS data into routes and activities (sections of a route, covered with a single means of transport), in case different means of transport are used in the same route;

4) effectiveness in identifying the means of transport;

5) low battery power consumption, allowing usual phone usage during the day, recharging the phone over the night;

6) near real-time, automated accessibility to the collected location data (via an application programming interface, i.e., an API).

Several fitness tracker apps (e.g., Moves, Nike Fuel or Runkeeper ${ }^{4}$ fulfill most of the above requirements and provide an API to retrieve location-tagged routes and activities performed by users. An internal testing of these apps showed promising results by Moves: it can identify three fitness activities with high accuracy (walking, running and cycling), which correspond to two means of transport (foot and bicycle; detected with an accuracy between $0.9-0.98$ ). More importantly, it also recognizes generic "transport" routes and activities performed during the day. Finally, battery power consumption is optimized, especially for iOS operating systems. However, during internal testing we also discovered several weaknesses:

- Moves' performances in tracking GPS activities are quite different depending on the smartphone operating system and device model: the best tracking performances are produced with recent iOS versions (from iPhone 5 onwards), followed by Android phones endowed with Internet mobile data connection; poorer performances are obtained with Android phones without data connection. Also, the accelerometer sensor offered by some phones is not compatible with Moves, which even happens with recent phone models;

- since Moves runs on a regular smartphone and tries to consume as little battery power as possible, it only records locations at irregular intervals. This implies that most activities are only mapped as beelines between the starting and the arrival point and are not map-matched in order to follow roads and railways. This can confuse

\footnotetext{
4 www.moves-app.com www.nikeplus.com runkeeper.com
}

users, especially when parts of routes go across lakes or mountains, and estimates incorrect values for the distances travelled;

- inter-modal routes are sometimes not correctly detected, being instead classified as a single-mode route. Surprisingly, this even takes place with the same smartphone on the same route, though on different days. The most likely reason is that Moves does not record a tracking point at the moment of mode change (due to a low GPS points tracking frequency) and thus can not split up the route in different modes. Alternatively, this might be a weakness of the algorithms used by Moves to detect modal changes;

- the precision of visited places is sometimes inaccurate, which makes it difficult to infer the reason for a trip, such as going shopping, or going to the gym.

Despite these limitations, we opted for Moves since it fulfilled the majority of requirements indicated above, especially points 2), 3) and 4) (although limited to walking, cycling and motorized transport), which the other apps we examined could not guarantee. A big challenge in using Moves to identify mobility patterns remains the correct identification of the means of transport associated with activities classified by Moves as "transport" (i.e., distinguishing between taking the bus, the train, subway or cars). And, of course, accuracy of the data gathered by Moves is affected by limitations in the overall quality of the GPS network signals or by wrong settings in the users' smartphones, such as disabling location services. This, however, would have happened with any other app and it is not dependent on Moves.

\section{Challenges}

Opting for exploiting Moves data via its API leads to one big issue: it is not possible to modify the tracker app nor the related data tracking processes and algorithms. The main consequences are the following ones:

1) To allow users to easily interact with the collected data, they should be able to access data directly via the smartphone, instead of for example via a reserved area on the project website. Users therefore need to install two apps (in our case, the fitness app Moves and the GoEco! Tracker app) and to connect them.

2) Tracking accuracy, retrieved information, battery consumption and availability on different operating systems are out of direct control.

3) The retrieval and updating of tracking data leads to increased complexity and causes a delay in data processing, because data must be fetched via the API.

4) Data are inevitably stored also on third-party servers, which might be in another country and subject to different data protection laws.

The first item can build an initial barrier for people to participate, not only because it increases the complexity of installing the mobility tracking app, but also because it forces users to create multiple accounts. This requires detailed documentation of the setup process and communication of the 
reason for using a multi-application setup. However, of all the 130 support requests we received by email during the first four weeks of the GoEco! monitoring periods, only 3 were directly concerned with the multi-app setup. As such, users do not seem to be restricted by the multi-app setup.

Of the 638 people who originally signed up for the GoEco! living lab experiment, 41 had operating systems which were not compatible with the tracker app (mostly Windows Phone and older versions of iOS). We tried to keep this number low by communicating that the study participants must either own an iPhone or Android smartphone. It is interesting to note that only 19 out of these 41 (approx. 50\%) actually informed us that they could not participate in the project due to incompatibilities. The others either did not try, or gave up without giving notice. While remarks on battery issues can be found in several support requests, only one participant explicitly left the project because of it. Finally, 31 support requests (approx. 25\%) concerned bad tracking quality and missing activities. Though only two users left the study for this reason, it shows that refinement of tracked data is necessary.

The communication via the tracker API leads to additional technical difficulties. First of all, the tracker app credentials have to be stored centrally, and mechanisms have to be in place to ensure that accounts are always correctly linked, even if someone re-installs an app, switches a phone or loses login information. Second, the communication causes a delay in processing the activities. While some APIs provide a push notification when new activities are available, fetching and processing them still causes a considerable delay. This forced us to promote that users should interact with the app on a daily basis, instead of after every activity they completed. While this is acceptable for mere data collection, many user interactions rely on well-timed feedback, and are thus out of reach for such an application setup [15].

While we only received two support requests concerning privacy, both participants stating that they leave the experiment, it was one of the most discussed points during preparation of the project. Because all the data is stored in at least two places now, one of which is owned by the commercial company owning Moves, and because location traces are generally considered sensitive data [41], [42], proper communication is necessary: we explicitly dealt with privacy protection issues both on the project website and during recruitment meetings. We suspect, however, that most people, who worried about privacy, did not sign up for the experiment in the first place. Figure 1 shows the most mentioned reasons for leaving the project. The most prevalent was an unsupported operating system by Moves on older iPhones. Even though privacy questions cover a significant part of all support requests, not many people left the experiment because of it.

The considerations above led us to design the general architecture of the GoEco! mobility tracking system as represented in Figure 2 2. a user installs the Moves app and keeps it active in background on her phone, without interacting with it. She also installs the GoEco! Tracker app, with which she interacts on a daily basis. As soon as GoEco! Tracker sets up

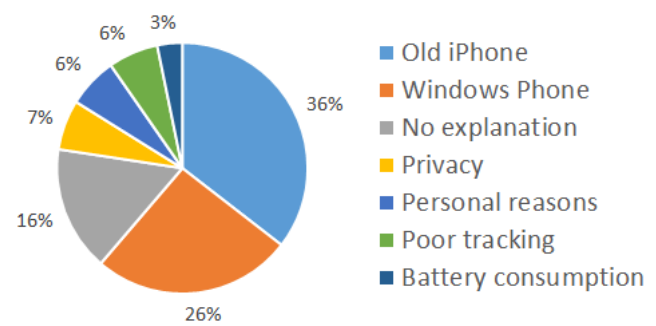

Fig. 1. Reasons for Leaving the GoEco! experiment.

communication with Moves, all the travelled routes recognized by Moves are also recorded and stored on the GoEco! server. In particular, the server receives a notification from Moves when new routes are available, fetches them via the API, calculates route refinements and stores them in a database. GoEco! Tracker displays these routes to the user upon the next interaction, and offers the possibility to check and validate them. Finally, the updated routes are again stored on the GoEco! server.

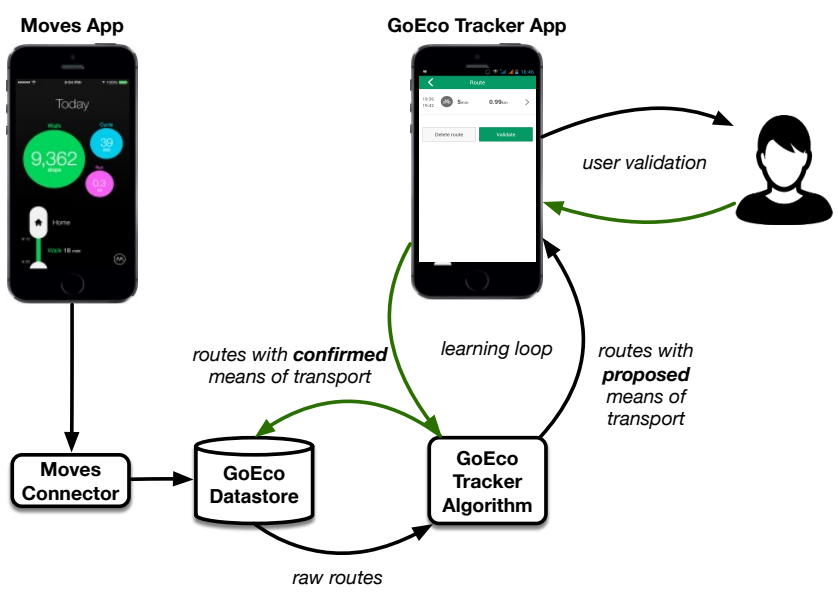

Fig. 2. Architecture of the GoEco! Tracker system.

As already indicated, the locomotion tracking in Moves only covers fitness-related activities, i.e., there is no distinction between transport modes such as the car or the train. Because it is not possible to influence the received information, we have to introduce an additional step that infers the transport mode from the information given by Moves (a detailed description is given in section $\mathrm{VI}$. To check the accuracy of this additional step, and to ensure valid data, users have to validate every activity they perform: the GoEco! Tracker app asks them to check the path they traveled, verifying the start and destination point of each activity, the time of the day and the related means of transport. In the case of wrong data, users can either specify the correct means of transport, or, if they do not acknowledge having traveled such a route, they can remove the route from the dataset. While this provided us with corrected data, it was also the source of most support requests and uncertainty: often, recorded activities are not perfectly accurate, which makes users wonder when they should validate an activity, or when 
to discard it. Of all the 52'985 activities collected by GoEco! during the study period from March 7 to April 4 2016, a total of $37^{\prime} 510$ were validated by the study participants $(70.8 \%)$.

\section{IDENTIFICATION OF THE TRANSPORT MODE}

As already explained, Moves classifies the recorded activities into walking, running, cycling and motorized transport. In order to correctly identify the users' mobility patterns, GoEco! Tracker has to consider a more refined list of means of transports (MoT): foot (including both walking and running), bike, electric bike, kick scooter, car, electric car, motorbike, scooter, bus, train, tram, plane, and ship. To prevent users from having to manually enter transport modes, GoEco! Tracker suggests a MoT for every activity, inferring it from activity data (such as time, start and end point, etc.) and from the history of user activities. Because a user only has to manually correct the MoT when the app suggestion is incorrect (Figure 3 shows the validation screenshots), achieving a high accuracy in the identification of the MoT is essential.

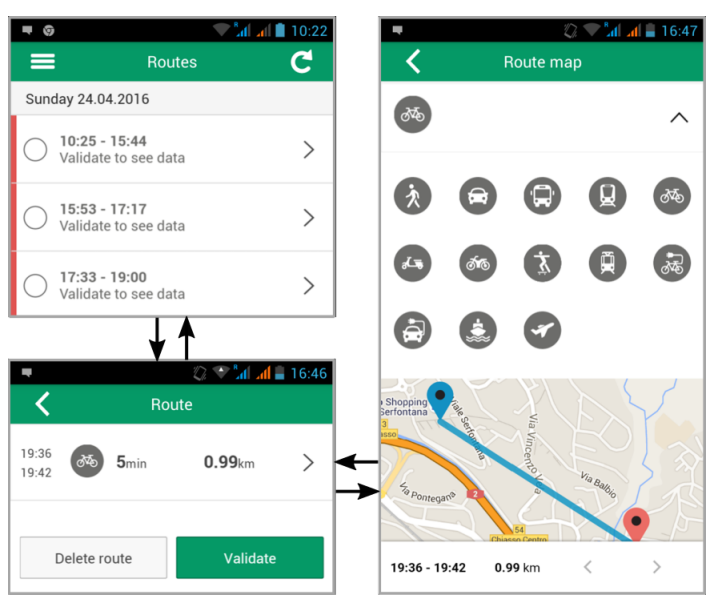

Fig. 3. The screenshots for the MoT validation in GoEco! Tracker.

The data recorded by Moves for each activity and available to GoEco! Tracker include the traveled distance, the duration of the activity, the start and arrival times and GPS coordinates, and the time and coordinates of an arbitrary number of intermediate track-points (arbitrary in the sense that Moves does not provide information about the criteria based on which a position along an itinerary is recorded as a track-point).

As stated in Section III a large body of research is devoted to the identification of the MoT based on data from smartphones. However, most of it relies on a richer set of data than the one that can be extracted from Moves records. In [43], a very large accuracy of .985 is obtained using GPS data and accelerometer measurements which are not available from Moves (notice moreover that in [43] only foot, bike and motorized transport are discriminated). Other work, such as [21], [44], [45], [46], considers more MoTs, reaching accuracies ranging from .771 up to .937, however, they only consider a maximum of 5 MoTs. Moreover, all use high frequency GPS data from which motion features such as acceleration and instantaneous heading change can be derived. The extraction of such features is made difficult in our case by the lack of a continuous collection of GPS data imposed either by battery saving requirements or by limitations in the quantity of the stored information on Moves servers. Note that the high accuracy of .937 obtained in [21] is reached using, besides motion features, the matching of GPS data with the transportation network and with the real time bus location, the latter being hardly available in most applications. However, also other works, e.g., [46], [45], show that map matching has the potential for improved classification accuracy.

To tackle the challenging classification task posed by the GoEco! setup, we build upon results from above research, but also consider information that can be drawn from the transportation network. In particular, we use the Open Trip Planner (OTP) system [47] (an open source and open data transport routing system, supporting a variety of modes, such as walking, taking public transport, driving, or biking 5 to identify possible connections from the start to the destination point of each activity using public transportation (PT). More precisely, for each activity we query the OTP to obtain the best transfer solution by bus only, train only and tram only (this includes walking to the respective PT stop). This allows us to see if it is actually possible to perform the activity using public transport, and, if possible, which was the most likely used mode. Ultimately, the features considered are:

- $f_{1}=$ average speed $(\mathrm{km} / \mathrm{min})$. It mainly discriminates plane, other motorized transport modes, bike and foot.

- $f_{2}=$ total traveled distance $(\mathrm{km})$. Discriminates approx. the same as $f_{1}$.

- $f_{3}=$ maximum distance between track-points over the traveled distance. It is included because track points of activities such as plane and train are often very sparse in some sections along the itinerary.

- $f_{4}=$ average heading change between track-points. The heading change is computed as the change of heading direction between two consecutive track-points. The use of this feature is motivated by the fact that, being constrained by a road, people driving a motorized MoT cannot change their heading direction as flexibly as people walking or cycling [44]. Unfortunately, this feature is less informative in our setting, because Moves does not record track-points at a very high frequency.

- $f_{5}, f_{6}, f_{7}=$ difference between the actual duration of the travel and the duration (waiting time excluded) of the solutions suggested by the OTP by, respectively, train, bus and tram.

- $f_{8}, \ldots, f_{13}=$ distance of the start and arrival points of the recorded itinerary from the departure and arrival stops of the OTP suggested solutions by train, bus and tram.

- $f_{14}, f_{15}, f_{16}=$ number of stops of the OTP solution (by train, bus and tram) which are closer than 50 meters to one of the itinerary track-points, divided by the total traveled distance.

\footnotetext{
5 http://www.opentripplanner.org
} 
We can formalize this problem as a classification problem where each activity is an instance described by the vector features $\mathbf{f}=\left[f_{1}, \ldots, f_{16}\right]$ and belonging to the unknown class $c_{a}$ among the set of classes $c_{j}, j=1, \ldots, 13$ representing all possible MoTs, that is, $c_{j}=\{$ foot, bike,..., ship $\}$. In order to improve the classification accuracy, we account for the different mobility patterns of individual users at two levels:

- we account for the different distribution of the mobility patterns of different users. For instance, the distribution of distances traveled by "car" activities can differ between users in large cities and users in small towns.

- we account for the routines of the different users by considering the specific means of transports historically used by the user for the same itinerary.

The different distribution of mobility patterns is accounted for by training a different classifier for each user. As there are no training data available for the users involved in GoEco! prior to the beginning of the mobility tracking activities, the different classifiers must be learned online. With this in mind, we adopted a naive Bayes (NB) classifier, since its parameters can be sequentially updated as new validated data become available from the tracking activity. The NB classifier assigns to each class $c_{j}$ the probability $P\left(c_{j} \mid \mathbf{f}\right)$ based on the Bayes rule and the assumption of independence of the different features:

$$
P\left(c_{j} \mid \mathbf{f}\right)=\frac{P\left(f_{1} \mid c_{j}\right) P\left(f_{2} \mid c_{j}\right) \cdots P\left(f_{m} \mid c_{j}\right) P\left(c_{j}\right)}{p(\mathbf{f})} .
$$

where the probabilities $P\left(c_{j}\right), j=1, \ldots, 13$ represent the prior opinion about which means of transport is more likely to be used, whereas the probabilities $P\left(f_{i} \mid c_{j}\right), i=$ $1, \ldots, 16, j=1, \ldots, 13$ represent the conditional probability of observing the value $f_{i}$ for the $i$-th feature given that the instance belongs to the $j$-th class. As a result of this inference process, we obtain an estimate of the probability of each MoT for the activity to be classified. Finally, to account for the user routines, we consider all the previous activities where the user has traveled the same itinerary. Two activities are assumed to correspond to the same itinerary whenever the distances between their start and destination positions are both smaller than 150 meters. Let $n$ be the number of historical segments of a user which correspond to the same itinerary of the activity to be classified, and $n_{j}$ the number of activities among them belonging to class $c_{j}$. The probability

$$
P\left(c_{j} \mid \text { itinerary }\right)=\frac{n_{j}+\alpha}{n+13 \alpha},
$$

where $\alpha$ is a smoothing parameter, provides an estimate of the probability that the $j$-th mean of transport is used by the current user on the current itinerary. Then, to account for the user routines, we re-calculate the probability estimates $P\left(c_{j} \mid \mathbf{f}\right)$ of each class $c_{j}$, issued by the NB classifier, as follows:

$$
\hat{P}\left(c_{j} \mid \mathbf{f}\right)=\frac{1}{K} P\left(c_{j} \mid \mathbf{f}\right) P\left(c_{j} \mid \text { itinerary }\right)
$$

where $K$ is a normalizing constant. The activity is then assigned the MoT with the maximum post-processing probability $\hat{P}\left(c_{j} \mid \mathbf{f}\right)$.
Concerning the parameters of the NB classifier, features $i=1, \ldots, 4$ are assumed to have a Gaussian probability distribution $P\left(f_{i} \mid c_{j}\right)$. For all other features, we have to consider also the case of missing data, which happens when the OTP is not able to suggest any solution. Missing data can be very informative about the MoT used; for instance, if a solution by train cannot be found by the OTP for given start and destination positions, then, most likely, the corresponding itinerary has not been travelled by train. Therefore, we consider the boolean variable OTP (=true if an OTP solution was found, $=$ false otherwise) as a further feature and model the conditional probability $P\left(f_{5}, \ldots, f_{16}, O T P \mid c_{j}\right)$ as follows

$P\left(\mathbf{f}_{5: 16}, O T P=0 \mid c_{j}\right)=P\left(O T P=0 \mid c_{j}\right)$

$P\left(\mathbf{f}_{5: 16}, O T P=1 \mid c_{j}\right)=P\left(O T P=1 \mid c_{j}\right) P\left(\mathbf{f}_{5: 16} \mid c_{j}, O T P=1\right)$

where $P\left(\mathbf{f}_{5: 16} \mid c_{j}, O T P=1\right)=\prod_{i=5}^{16} P\left(f_{i} \mid c_{j}, O T P=1\right)$ is the conditional distribution of features $f_{i}, i=5, \ldots, 16$ given that an OTP solution has been found, and $P\left(O T P=1 \mid c_{j}\right)$ (resp. $P\left(O T P=0 \mid c_{j}\right)$ ) is the probability of finding (resp. not finding) an OTP solution when the mean of transport is $c_{j}$. The conditional distribution $P\left(f_{i} \mid c_{j}, O T P=1\right)$ of features $i=5, \ldots, 16$ is assumed to be Gaussian.

Within a Bayesian framework, a prior for the parameters of $P\left(f_{i} \mid c_{j}, O T P=1\right), i=1, \ldots, 16$ is assumed for each feature and class based on set of historical data collected from a small set of users involved in the project. Then, as a new set of validated mobility records $\mathbf{X}_{u}$ is collected for the $u$-th user, the prior is updated, by the Bayes rule, obtaining the posterior predictive distribution $P\left(f_{i} \mid c_{j}, \mathbf{X}_{u}\right), i=1, \ldots, 16$. Note that this updating procedure can be performed sequentially, that is, when a new record $x$ is collected, the posterior obtained in the previous step $P\left(f_{i} \mid c_{j}, \mathbf{X}_{u}\right)$ is assumed as the new prior and updated with the new record $x$. Moreover, by exploiting the properties of conjugate priors the online updating procedure is fast and simple. Therefore, we have assumed the normalGamma prior on the parameters of $P\left(f_{i} \mid c_{j}\right), i=1, \ldots, 4$, and $P\left(f_{i} \mid c_{j}, O T P\right), i=5, \ldots, 16$ and the Beta prior for $P\left(O T P \mid c_{j}\right)$.

By learning the users' mobility patterns, we have been able to improve the performance of the classification algorithm during the tracking period, thus requiring the users to interact with the app less and less, as time goes by.

Preliminary assessments of the overall performances of the algorithm are related to one month of use of the GoEco! Tracker app by 359 study participants (from March 7 to April 4, 2016): we compare the MoT proposed by the app and the MoT recorded after validation by the user. Figure 4 shows the evolution of the classification accuracy for each MoT $m$ (defined as the number of activities correctly classified as $m$ divided by the total number of activities collected for $m$ ) as a function of the number of training activities $n_{u, m}$ (that is, activities previously collected and validated) available for user $u$ and MoT $m$. Only the 7 MoTs used by at least 5 users for more than 15 times are shown in the Figure. The accuracy is 
averaged over all users and over a window of 15 values of $n_{u, m}$, that is from $n_{u, m}$ to $n_{u, m}+14$.

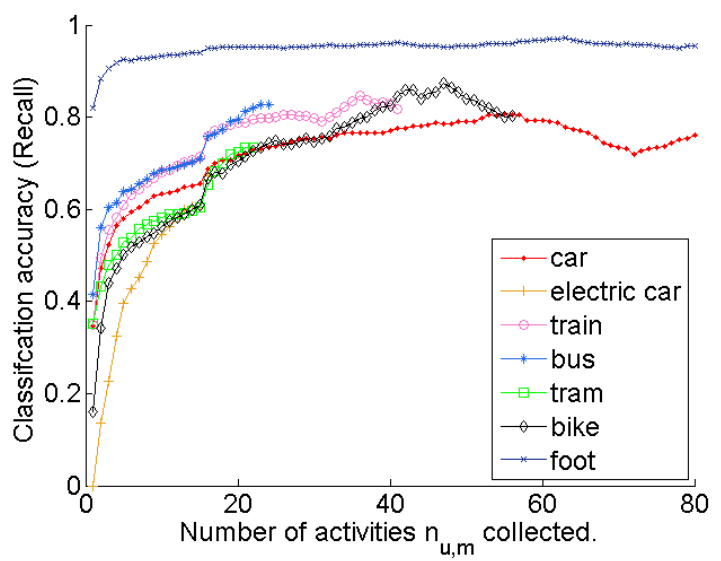

Fig. 4. Evolution of the classifier accuracy as more data are collected for each user.

We can notice that the classification accuracy of "foot" is very large. This is mostly due to the low speed and small distances typically travelled by foot, which make this MoT easy to classify. Notice that as $n_{u, m}$ (the x-coordinate in Figure (4) increases, the number of users included in the analysis reduces. Therefore, the weight of a single user on the overall performance increases towards the end of the curves in figure 4, causing the oscillation in the performance that can be observe, e.g., for bike and car. Indeed, the classification performance varies among users, and thus a single user for which the performances are particularly poor or particularly good can significantly affect the average performance when only few users are considered.

Table II shows the accuracy achieved from the beginning of the study and that obtained when classifying only those activities corresponding to a user and a MoT for which at least $n_{u, m}=25$ training activities were collected. The overall classification accuracy is satisfactory in both cases (.829 in the former case and .891 in the latter), also because of the weight of walking activities which can be classified with high accuracy (and are, in most cases, already correctly classified by Moves). On the other side, when considering only the classifications for which sufficient training data were available, an acceptable accuracy of more than .750 is obtained for all MoTs except for the electric bike (which can be hardly distinguished from a traditional bike) and the bus (which is hard to discriminate from the car).

These results could be further improved by considering a hierarchical model for deriving the classifier parameters, that is, a model that assumes a common distribution over the parameters of the different user classifiers. Such hierarchical approach has the capability of recognizing clusters of similar users in an unsupervised way, and learn their common features from their joint datasets. Therefore, it is expected to allow exploiting similarities between user to improve estimates of

\begin{tabular}{ccc} 
MoT & \multicolumn{2}{c}{$\begin{array}{c}\text { Accuracy } \\
\text { Activities with } n_{u, m} \geq 25\end{array}$} \\
\hline Foot & .9530 & .9579 \\
Bike & .7037 & .8386 \\
Electric bike & .4715 & .6071 \\
Kick scooter & .4508 & - \\
Car & .7252 & .7633 \\
Electric car & .7362 & .8162 \\
Motorbike & .5867 & .8750 \\
Scooter & .3765 & .9500 \\
Bus & .7288 & .7120 \\
Train & .7459 & .8155 \\
Tram & .6464 & .7778 \\
Plane & .6531 & - \\
Ship & 0 & - \\
Overall & .8294 & .8909
\end{tabular}

CLASSIFICATION ACCURACY OVER ALL ACTIVITIES AND OVER THE ACTIVITIES CORRESPONDING TO A USER AND A MOT FOR WHICH $n_{u, m} \geq 25$.

the classifier parameters, while still tailoring each classifier to the individual user. Also, a more intense exploitation of map matching methods, besides those within the OTP routing system, could result in an increased classification accuracy.

\section{AnAlyzing TRACKed DATA}

In this section we perform an analysis of activity data collected by one month of large scale testing of the GoEco! Tracker app. We look at a set of key measures, concerning distances covered, and transport modes involved. From the 461 users who registered in the app, a total of 359 collected more than one activity during the GoEco! experiment period from March 7 to April 4 2016. Of them, 292 users started data collection within the first five days of the study period. This means, they set up the connection between the Moves and GoEco! Tracker apps, and left it active for at least one route. Figure 5 shows the distribution of days on which users tracked activities. There is a considerable number of users who only provided activities (i.e., collected them by activating Moves and submitted them to the system by connecting Moves to GoEco! Tracker) on a small number of days.

Those participants either left the project early or downloaded the app very late in the study period. Because they do not provide enough data, we will from now on only consider the 199 participants who collected and submitted activities on 21 or more days over the four weeks monitoring period (cf. the red dotted line in Fig. 5. We could also consider only the 142 users who validated activities on 21 or more days (which would give us the most accurate dataset), however, in a first step, it is interesting to also look at the amount of users who did not perform the validation step, and how we might still extrapolate their tracked activities. We will lead an indepth investigation on why participants were not as active as expected in GoEco! mobility tracking activities later during the project, by means of interviews and focus groups with a selection of users.

On average, we collected 7.4 activities per user per day, of which $77.1 \%$ were actively validated (31'782 out of 41'199). Figure 6 shows the total number of activities, with their 


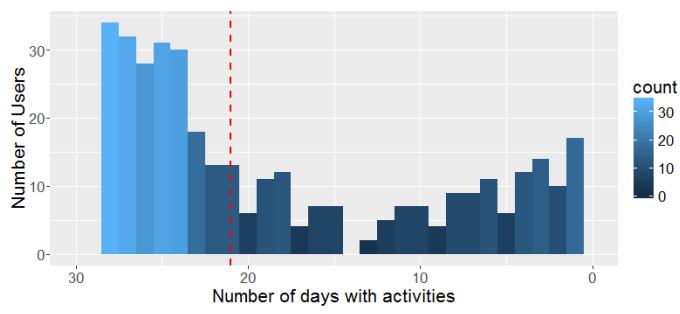

Fig. 5. Number of days on which users collected at least one activity.

respective mode of transport, collected in the four weeks period. A large number of activities is of type walking (38.4\%), which results from the fact that the Moves fitness tracker even records very short walking routes (around hundred meters), because it is not primarily interested in mobility behavior, but in everything that contributes to health. However, due to the fact that these routes are very short, they do not contribute much to overall mobility and could easily be filtered out if desired, for example when determining the modal share of the GoEco! participants. Another large part is made up of unknown routes $(22.9 \%)$. In our setting, those are routes which have not been validated by users. Using the method described in section VI. we could also assign a mode of transport to all of them.

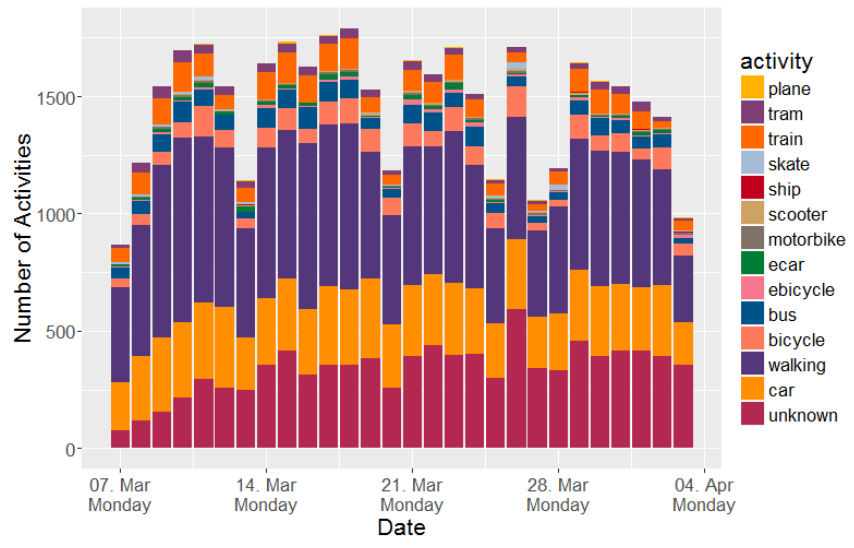

Fig. 6. Activities Collected by GoEco! Tracker During Four Weeks.

The primary reason for the increase of unknown routes is the declining novelty of the app: users start neglecting the manual validation in GoEco! Tracker, while they still keep Moves tracking in the background. We found that most users either only validated their routes a couple of days, or the whole period. As such, we can also filter out people who do not validate, but just let themselves be passively tracked.

Looking at the graph of activities in 6 two further characteristics stand out: the weekly periodicity, which can be seen by comparing at the average number of activities performed on weekdays with those on Sundays, and the weekend of the 25th to the 27th of March. While we collected an average of 1'532 activities per day on weekdays, this number drops to 1'092 on Sundays (-28.7\%). This looks reasonable, considering that most of the regular trips (such as going to work) are not performed on Sundays. However, it is interesting to note that there is no big difference between weekdays and Saturdays, even though most people have this day off as well. This is most likely due to the fact that shops are open (as in contrast to Sundays), which allows people to perform a wider range of activities. The weekend around 27th was Easter holiday, when Friday and Monday are days off (and shops are closed) in Switzerland. As such, the number of activities drops to comparable levels to those of Sundays. Note that in the analysis so far, we did not look at distances covered, but only at the number of activities.

To get an idea of the accuracy of using the Moves fitness tracker app to measure mobility behavior, we perform a comparison with data from the Swiss Mobility and Transport census [1]. A first measure to consider are the totally traveled kilometers. Figure 7 shows the average distance covered by study participants on every day. We manually removed 43 plane trips, as they led to large outliers on single days. The overall average $(56.2 \mathrm{~km}$; the red dotted line) is about 15 $\mathrm{km}$ higher than the one indicated by the mobility census (a yearly total of $15^{\prime} 246 \mathrm{~km}$, or $41.8 \mathrm{~km}$ per day, also excluding plane journeys; the black dotted line). While this does not exactly match, it does not necessarily reflect errors in tracking accuracy, but it is most likely induced by our sample, which consists of people interested in optimizing travel behavior, probably because they travel a lot.

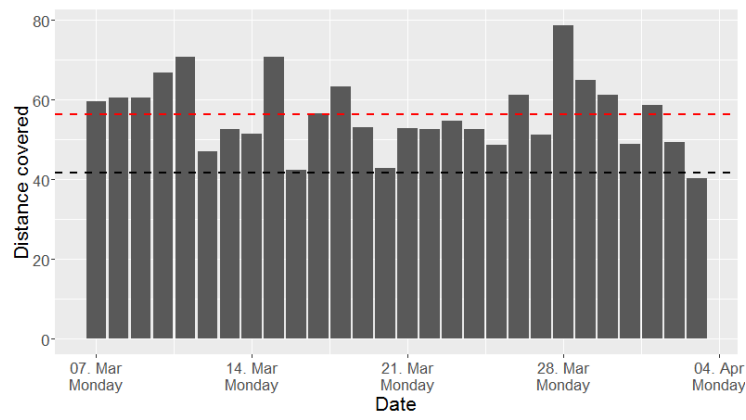

Fig. 7. Kilometers Traveled by Users, by day.

The second key measure concerns the average distance covered by each mode of transport. This is a bit more difficult, considering our dataset contains a number of unknown activities. For this analysis, we replaced unknown activities with the one discovered using the method described in Section VI Figure 8 shows the resulting distribution of modes, and the number of kilometers traveled with each. Again, we manually removed the plane journeys, and also summed trams and buses (category "tram" in the figure), cars and e-cars, and bicycles and e-bicycles (because the respective categories were summed in the Swiss mobility census as well). Finally, we are not showing the other MoT categories because the distances covered are negligible. As can be seen, the largest difference respect to the Census is due to an higher use of trains, trams and buses. This might be explained by the fact that a large part of our sample is commuting to the city of Zurich, a journey 
usually covered by train.

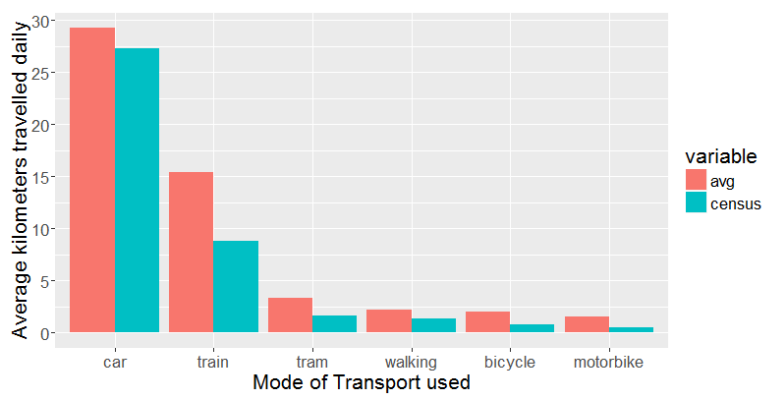

Fig. 8. Modes Used for Kilometers Traveled

Summarizing, while the data tracked by Moves and inferred by our algorithm from Section $\mathrm{VI}$ are not perfectly accurate, they are in acceptable ranges. Problems like beeline routes, or non-validated (i.e., unknown) routes can be handled using map-matching and our own classifier. It remains to be seen if the data is accurate enough to provide users with viable alternative routes (or even to match their needs [48]), which Gamification elements can be employed, and whether modal shifts are large enough to be detectable. The next study period of GoEco! should be able to give an answer to these questions.

\section{CONClusions AND Discussion}

We presented our experiences using the Moves fitness tracker app for mobility tracking. The reasons to use an existing app are obvious: a widely available, tested application that already provides first data analysis. Among the risks and challenges we faced were the need to install two applications, with included technical overhead, privacy issues, the risk that the tracker application would have been disabled during the study and that we completely had to rely on the location and activity data provided by the Moves tracking application, without direct control over them. However, we showed that most issues proved to be minor problems and the collected data are of high accuracy and can be analysed with respect to different aspects. Most people who left the GoEco! living lab did it due to unsupported operating systems and devices or generally because they lost interest in the project.

Two key issues are to be considered to further improve data quality and face the lack of precision we registered on some devices. First: Moves can miss all intermediate points of a route and simply store the start and arrival points. In those cases, computation of the distance travelled is based on a beeline. This may result in a very rough estimate affecting all subsequent calculations about mobility patterns and related energy consumptions and $\mathrm{CO}_{2}$ emissions. Therefore, we suggest to correct this information by searching for the best map matching between the road and public transport networks and the activity defined by its start and arrival positions and by the (known) mean of transport used. This would allow a better match between activities and routes actually taken, also favouring perceptions of accuracy in the eyes of the users.
Second: sometimes Moves fails to recognize modal changes and incorrectly splits multimodal routes. Thus, the same activity includes parts of a route travelled with two different means of transport. For example, it may miss a change from tram to bus and show the whole route as a single activity by motorized transport. This is an error that cannot be corrected by the user in the validation activities performed in GoEco! Tracker, since we opted for keeping the user interface as simple as possible and for limiting interactions with the users. However, by learning users routines, in future versions of GoEco! Tracker it could be possible to correct at least the activities that match users' systematic multimodal routes. Also, map matching strategies, such as those used in [21], could be used to learn reference places where a mode change is particularly likely. Having recorded their position, the modal change points missed by Moves inside a route could be identified by comparing its trackpoints with those of the reference places.

Among the improvements we will introduce in the future is the identification of car-pooling and car-sharing means of transport. Correctly tracking their use will allow us to estimate even more realistic consumption and emission patterns and also to monitor the ongoing societal transition from owning private means of transport to using transport services. Finally, in parallel to improving the overall performances of the GoEco! Tracker app, we are now entering the second phase of the GoEco! project, when the experience will be gamified in order to nudge people to change their mobility patterns, towards an overall reduction in the use of the car and the related energy consumptions and $\mathrm{CO}_{2}$ emissions.

\section{ACKNOWLEDGMENTS}

This research was supported by the Swiss National Science Foundation (SNF) within NRP 71 "Managing energy consumption" and by the Commission for Technology and Innovation (CTI) within the Swiss Competence Center for Energy Research (SCCER) Mobility.

\section{REFERENCES}

[1] B. für Statistik and B. für Raumentwicklung, "Mobilität in der schweiz, ergebnisse des mikrozensus mobilität und verkehr 2010," Mobililität und Verkehr. Schweiz, S. d. Neuchâtel und Bern, Bundesamt für Statistik, pp. $1-120,2012$.

[2] S. Handy, B. Van Wee, and M. Kroesen, "Promoting cycling for transport: Research needs and challenges," Transport reviews, vol. 34, no. 1, pp. 4-24, 2014.

[3] B. fr Statistik (BfS). (2014) Informationsgesellschaft gesamtindikatoren / infrastrukturen - telefoninfrastruktur. [Online]. Available: http://www.bfs.admin.ch/bfs/portal/de/index/themen/16/04/ key/approche_globale.indicator.30101.1.html

[4] L. Montini, S. Prost, J. Schrammel, N. Rieser-Schüssler, and K. W. Axhausen, "Comparison of travel diaries generated from smartphone data and dedicated gps devices," Transportation Research Procedia, vol. 11, pp. 227-241, 2015.

[5] K. T. Geurs, T. Thomas, M. Bijlsma, and S. Douhou, "Automatic trip and mode detection with move smarter: First results from the dutch mobile mobility panel," Transportation Research Procedia, vol. 11, pp. 247-262, 2015.

[6] H. Becker, F. Ciari, M. Brignoni, and K. W. Axhausen, "Impacts of a new free-floating carsharing system traced with a Smartphone App." International Association for Travel Behaviour Research (IATBR), 2015. 
[7] P. Weiser, S. Scheider, D. Bucher, P. Kiefer, and M. Raubal, "Towards sustainable mobility behavior: Research challenges for location-aware information and communication technology," GeoInformatica, pp. 1-27, 2015.

[8] T. R. Dillahunt and J. Mankoff, "Understanding factors of successful engagement around energy consumption between and among households," in Proceedings of the 17th ACM conference on Computer supported cooperative work \& social computing. ACM, 2014, pp. 1246-1257.

[9] C. Fischer, "Feedback on household electricity consumption: a tool for saving energy?" Energy efficiency, vol. 1, no. 1, pp. 79-104, 2008.

[10] J. Froehlich, T. Dillahunt, P. Klasnja, J. Mankoff, S. Consolvo, B. Harrison, and J. A. Landay, "Ubigreen: investigating a mobile tool for tracking and supporting green transportation habits," in Proceedings of the SIGCHI Conference on Human Factors in Computing Systems. ACM, 2009, pp. 1043-1052.

[11] S. Gabrielli, R. Maimone, P. Forbes, and S. Wells, "Exploring change strategies for sustainable urban mobility," in Designing Social Media for Change at the ACM SIG-CHI Conference on Human Factors in Computing Systems (CHI 2013), 2013.

[12] A. Jylhä, P. Nurmi, M. Sirén, S. Hemminki, and G. Jacucci, "Matkahupi: a persuasive mobile application for sustainable mobility," in Proceedings of the 2013 ACM conference on Pervasive and ubiquitous computing adjunct publication. ACM, 2013, pp. 227-230.

[13] J. Bie, M. Bijlsma, G. Broll, H. Cao, A. Hjalmarsson, F. Hodgson, P. Holleis, Y. van Houten, K. Jacobs, J. Koolwaaij et al., "Move better with tripzoom," International journal on advances in life sciences, vol. 4, no. 3/4, 2012

[14] J. Hamari, J. Koivisto, and T. Pakkanen, "Do persuasive technologies persuade?-a review of empirical studies," in Persuasive Technology. Springer, 2014, pp. 118-136.

[15] P. Weiser, D. Bucher, F. Cellina, and V. De Luca, "A taxonomy of motivational affordances for meaningful gamified and persuasive technologies," ICT for Sustainability (ICT4S), 2015.

[16] E. Shove, "Changing human behaviour and lifestyle: a challenge for sustainable consumption," Department of Sociology, University of Lancaster: Lancaster, UK, 2003.

[17] F. R. Klauser and A. Albrechtslund, "From self-tracking to smart urban infrastructures: Towards an interdisciplinary research agenda on big data," Surveillance \& Society, vol. 12, no. 2, p. 273, 2014.

[18] S. Reddy, J. Burke, D. Estrin, M. Hansen, and M. Srivastava, "Determining transportation mode on mobile phones," in Wearable Computers, 2008. ISWC 2008. 12th IEEE International Symposium on. IEEE, 2008, pp. 25-28.

[19] N. Schüssler, K. W. Axhausen et al., "Processing gps raw data without additional information," 2008.

[20] N. Kiukkonen, J. Blom, O. Dousse, D. Gatica-Perez, and J. Laurila, "Towards rich mobile phone datasets: Lausanne data collection campaign," Proc. ICPS, Berlin, 2010

[21] L. Stenneth, O. Wolfson, P. S. Yu, and B. Xu, "Transportation mode detection using mobile phones and gis information," in Proceedings of the 19th ACM SIGSPATIAL International Conference on Advances in Geographic Information Systems. ACM, 2011, pp. 54-63.

[22] P. Nitsche, P. Widhalm, S. Breuss, and P. Maurer, "A strategy on how to utilize smartphones for automatically reconstructing trips in travel surveys," Procedia-Social and Behavioral Sciences, vol. 48, pp. 1033 1046, 2012.

[23] J. Ythier, J. Walker, and M. Bierlaire, "The role of the social network and the usage of communication in travel behavior measured with smartphone data," Technical Report TRANSP-OR 120801, Transport and Mobility Laboratory, ENAC, EPFL, Tech. Rep., 2012.

[24] Y. Yuan, M. Raubal, and Y. Liu, "Correlating mobile phone usage and travel behavior a case study of harbin, china," Computers, Environment and Urban Systems, vol. 36, no. 2, pp. 118 130, 2012, special Issue: Geoinformatics 2010. [Online]. Available: http://www.sciencedirect.com/science/article/pii/S0198971511000652

[25] F. Cellina, A. Förster, D. Rivola, L. Pampuri, R. Rudel, and A. E. Rizzoli, Environmental Software Systems. Fostering Information Sharing: 10th IFIP WG 5.11 International Symposium, ISESS 2013, Neusiedl am See, Austria, October 9-11, 2013. Proceedings. Berlin, Heidelberg: Springer Berlin Heidelberg, 2013, ch. Using Smartphones to Profile Mobility Patterns in a Living Lab for the Transition to E-mobility, pp. 154-163. [Online]. Available: http://dx.doi.org/10.1007/978-3-642-41151-9_15

[26] J. Jariyasunant, M. Abou-Zeid, A. Carrel, V. Ekambaram, D. Gaker, R. Sengupta, and J. L. Walker, "Quantified traveler: Travel feedback meets the cloud to change behavior,' Journal of Intelligent Transportation Systems, vol. 19, no. 2, pp. 109-124, 2015.

[27] M. Keally, G. Zhou, G. Xing, J. Wu, and A. Pyles, "Pbn: towards practical activity recognition using smartphone-based body sensor networks," in Proceedings of the 9th ACM Conference on Embedded Networked Sensor Systems. ACM, 2011, pp. 246-259.

[28] D. Shin, D. Aliaga, B. Tunçer, S. M. Arisona, S. Kim, D. Zünd, and G. Schmitt, "Urban sensing: Using smartphones for transportation mode classification," Computers, Environment and Urban Systems, vol. 53, pp. 76-86, 2015.

[29] P. Widhalm, P. Nitsche, and N. Brandie, "Transport mode detection with realistic smartphone sensor data," in Pattern Recognition (ICPR), 2012 21st International Conference on. IEEE, 2012, pp. 573-576.

[30] Y. Chon, E. Talipov, H. Shin, and H. Cha, "Mobility prediction-based smartphone energy optimization for everyday location monitoring," in Proceedings of the 9th ACM conference on embedded networked sensor systems. ACM, 2011, pp. 82-95.

[31] J. R. Kwapisz, G. M. Weiss, and S. A. Moore, "Activity recognition using cell phone accelerometers," ACM SigKDD Explorations Newsletter, vol. 12 , no. 2, pp. 74-82, 2011.

[32] E. Dons, T. Götschi, M. Nieuwenhuijsen, A. de Nazelle, E. Anaya, I. Avila-Palencia, C. Brand, T. Cole-Hunter, M. Gaupp-Berghausen, S. Kahlmeier et al., "Physical activity through sustainable transport approaches (pasta): protocol for a multi-centre, longitudinal study," BMC public health, vol. 15, no. 1, p. 1, 2015.

[33] X. Li, G. Pan, Z. Wu, G. Qi, S. Li, D. Zhang, W. Zhang, and Z. Wang, "Prediction of urban human mobility using large-scale taxi traces and its applications," Frontiers of Computer Science, vol. 6, no. 1, pp. 111-121, 2012.

[34] B. Bergvall-Kareborn and A. Stahlbrost, "Living lab: an open and citizen-centric approach for innovation," International Journal of Innovation and Regional Development, vol. 1, no. 4, pp. 356-370, 2009.

[35] A. Irwin, Citizen science: A study of people, expertise and sustainable development. Psychology Press, 1995.

[36] C. Liedtke, M. Jolanta Welfens, H. Rohn, and J. Nordmann, "Living lab: user-driven innovation for sustainability," International journal of sustainability in higher education, vol. 13, no. 2, pp. 106-118, 2012.

[37] F. Bauer, Smartphone-Fitness-Apps: Einflussfaktoren auf Tracking und Methoden zur Verbesserung der Wegaufzeichnung im urbanen Raum. na, 2012.

[38] M. J. Handel, "mhealth (mobile health) - using apps for health and wellness,' EXPLORE: The Journal of Science and Healing, vol. 7, no. 4, pp. 256-261, 2011

[39] B. Millington, "Smartphone apps and the mobile privatization of health and fitness," Critical Studies in Media Communication, vol. 31, no. 5, pp. 479-493, 2014.

[40] F. Cellina, A. Förster, D. Rivola, L. Pampuri, R. Rudel, and A. E. Rizzoli, "Using smartphones to profile mobility patterns in a living lab for the transition to e-mobility," in Environmental Software Systems. Fostering Information Sharing. Springer, 2013, pp. 154-163.

[41] J. E. Dobson and P. F. Fisher, "Geoslavery," Technology and Society Magazine, IEEE, vol. 22, no. 1, pp. 47-52, 2003.

[42] M. Duckham and L. Kulik, "Location privacy and location-aware computing," Dynamic \& mobile GIS: investigating change in space and time, vol. 3, pp. 35-51, 2006.

[43] H. Xia, Y. Qiao, J. Jian, and Y. Chang, "Using smart phone sensors to detect transportation modes," Sensors, vol. 14, no. 11, pp. $20843-$ 20865,2014

[44] Y. Zheng, Q. Li, Y. Chen, X. Xie, and W.-Y. Ma, "Understanding mobility based on gps data," in Proceedings of the 10th international conference on Ubiquitous computing. ACM, 2008, pp. 312-321.

[45] H. Gong, C. Chen, E. Bialostozky, and C. T. Lawson, "A gps/gis method for travel mode detection in new york city," Computers, Environment and Urban Systems, vol. 36, no. 2, pp. 131-139, 2012.

[46] D. J. Patterson, L. Liao, D. Fox, and H. Kautz, "Inferring highlevel behavior from low-level sensors," in UbiComp 2003: Ubiquitous Computing. Springer, 2003, pp. 73-89.

[47] A. Byrd and D. Emory, "K. webb. open trip planner analyst: Open source, open data driven transportation network analysis. poster p136039, session 616 (open data in transportation)," in Transportation Research Board Conference 2012.

[48] D. Bucher, P. Weiser, S. Scheider, and M. Raubal, "Matching complementary spatio-temporal needs of people," in Online proceedings of the 12th international symposium on location-based services, 2015. 